\title{
Melatonin induces cell apoptosis in Mia PaCa-2 cells via the suppression of nuclear factor- $\kappa B$ and activation of ERK and JNK: A novel therapeutic implication for pancreatic cancer
}

\author{
WEIMIN LI ${ }^{1}$, JIANSHENG WU ${ }^{2}$, ZHIYIN LI ${ }^{1}$, ZHIBO ZHOU $^{3}, \mathrm{CHAO} \mathrm{ZHENG}^{4}$,

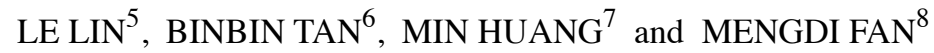

\begin{abstract}
${ }^{1}$ Department of Gastroenterology, The Affiliated Hospital of Hangzhou Normal University, Hangzhou, Zhejiang 310000; ${ }^{2}$ Department of Gastroenterology, The First Affiliated Hospital of Wenzhou Medical University, Wenzhou, Zhejiang 325000; ${ }^{3}$ Department of Infection, Zhejiang University International Hospital, Hangzhou, Zhejiang 310000; Departments of ${ }^{4}$ Endocrinology and ${ }^{5}$ Rehabilitation, The Second Affiliated Hospital of Wenzhou Medical University, Wenzhou, Zhejiang 325000; ${ }^{6}$ Department of Gastroenterology, Taizhou Municipal Hospital, Taizhou, Zhejiang 318000; ${ }^{7}$ Department of Obstetrics and Gynecology, The Second Affiliated Hospital of Wenzhou Medical University, Wenzhou, Zhejiang 325000; ${ }^{8}$ Department of Endocrinology, Zhejiang University International Hospital, Hangzhou, Zhejiang 310000, P.R. China
\end{abstract}

Received February 1, 2016; Accepted June 30, 2016

DOI: 10.3892/or.2016.5100

\begin{abstract}
Melatonin is synthesized by the pineal gland and is released into the blood. In the last several years, some studies have shown that melatonin has anticancer properties; however, the mechanisms behind the antitumour traits are unclear, especially in pancreatic cancer. Therefore, in the present study, we investigated the antitumour effects of melatonin on the human pancreatic carcinoma cell line MIA PaCa- 2 and explored its biological mechanisms. MIA PaCa-2 cells were treated with melatonin, and we used a CCK- 8 assay to evaluate the cell viability. We also used flow cytometry to observe cell apoptosis and western blot analysis to assess the protein expression. Our study found that melatonin inhibited cell viability, suppressed colony formation and reduced cell migration and invasion and induced cell apoptosis in MIA PaCa-2 cells. Our results showed that melatonin treatment inhibited $\mathrm{NF}-\kappa \mathrm{B}$ p65 activation. Moreover, melatonin treatment activated the mitogen-activated protein kinase pathways (c-jun $\mathrm{N}$-terminal kinase and extracellular-regulated kinase 1/2), which increased Bax protein expression and caspase-3 cleavage and decreased
\end{abstract}

Correspondence to: Dr Mengdi Fan, Department of Endocrinology, Zhejiang University International Hospital, 848 Dongxin Road, Xiacheng, Hangzhou, Zhejiang 310000, P.R. China

E-mail: 1wm900517@163.com

Abbreviations: MLT, melatonin; NF- $\kappa \mathrm{B}$, nuclear factor- $\kappa \mathrm{B}$; JNK, c-jun N-terminal kinase; MAPK, mitogen-activated protein kinases; ERK1/2, extracellular-regulated kinase $1 / 2$

Key words: pancreatic cancer, apoptosis, mitogen-activated protein kinases, melatonin, nuclear factor- $\mathrm{\kappa} \mathrm{B}$
Bcl-2 protein expression. These new developments demonstrate that melatonin plays a potential role in anticancer treatment and may act as an effective therapeutic agent in the future.

\section{Introduction}

It is estimated that approximately 277,000 cases of pancreatic cancer are diagnosed worldwide every year, accounting for $2.2 \%$ of all cancers (1). Pancreatic cancer is a multifactorial disease and the seventh leading cause of cancer-related death in China (2). Moreover, pancreatic cancer has lower rates of diagnosis and the 5-year survival rate is $<5 \%$ (3). Pancreatic cancer has limited treatment options. At present, surgery is considered as the only effective therapeutic measure. However, only $20 \%$ of the patients are suited for this approach (4). Therefore, more effective therapies are needed.

Melatonin is a pineal gland hormone and adjusts sleep and circadian rhythm (5). Melatonin also plays a part in biological processes including immunomodulation, antioxidative stress and anti-inflammation (6-9). Researchers have revealed that melatonin is an anticancer hormone (10). The anticancer mechanisms of melatonin involve activation of antioxidation stress, inhibition of migration and induction of cell apoptosis (11-13). Researchers showed that melatonin had an apoptotic effect on the hepatocarcinoma HepG2 cell line (14). Research has found that using melatonin has no significant side-effects (15). In conclusion, we postulated that melatonin may exert a protective effect against cancer.

The mitogen-activated protein kinase (MAPK) pathway plays an important role in cell survival and proliferation (16). The MAPK family has been categorized into three groups: c-jun N-terminal kinase (JNK), extracellular-regulated kinase $1 / 2(E R K 1 / 2)$ and p38MAPK. Some studies have suggested that the JNK substrate is involved in cell growth 
and apoptosis (17), which take part in tumor progression and are affected by melatonin (18).

Nuclear factor $-\kappa \mathrm{B}(\mathrm{NF}-\kappa \mathrm{B})$ of the Rel family includes p50, p52, p65 (RelA), c-Rel and Rel B. NF- $\kappa$ B, as a transcription factor, stimulates the expression level of many genes closely related to oxidative stress and anti-apoptosis (19). Tamura et al found that melatonin suppressed the NF- $\kappa \mathrm{B}$ pathway in rat endothelial cells (20). Gilad et al also revealed that melatonin suppressed the $\mathrm{NF}-\kappa \mathrm{B}$ pathway in murine macrophages (21).

At present, it is unclear whether melatonin has an effect on the apoptosis of the human pancreatic carcinoma cell line MIA PaCa-2 through the JNK and ERK pathways. Therefore, we examined the function of melatonin on the viability and apoptosis of MIA PaCa-2 cells via the MAPK signaling pathways and we investigated whether melatonin induces cell apoptosis through a decrease in $\mathrm{NF}-\kappa \mathrm{B}$.

\section{Materials and methods}

Cell culture and reagents. Human pancreatic carcinoma cell line MIA PaCa-2 was purchased from the Cell Bank of the Type Culture Collection of the Chinese Academy of Sciences (Shanghai, China). The cells were cultured in Dulbecco's modified Eagle's medium (DMEM) (Sigma, St. Louis, MO, USA) supplemented with $1 \%$ penicillin/streptomycin and $10 \%$ heat-inactivated fetal bovine serum (FBS; Sigma) in $5 \% \mathrm{CO}_{2}$ in a humidified incubator. Cells were seeded at a density of $5 \times 10^{5}$ cells/100-mm dish. Melatonin (Sigma) was dissolved in $0.2 \%$ dimethyl sulphoxide (DMSO) and cells were treated with different doses of melatonin (0-4 mM) from 0 to $72 \mathrm{~h}$.

Colony-forming assays. MIA PaCa- 2 cells were seeded into 6 -well plates $\left(5 \times 10^{5}\right.$ cells/well $)$ at $37^{\circ} \mathrm{C}$ with $5 \% \mathrm{CO}_{2}$ in a humidified environment. On the second day, the cells were treated with 1 and $2 \mathrm{mM}$ melatonin for 8 days. Then each well was washed twice with PBS and stained with crystal violet. During the incubation period of 8 days, the culture medium was replaced every 3 days in all wells.

Cell viability assay. The Cell Count Kit-8 (CCK-8; Dojindo, Japan) was used to evaluate the effects of melatonin on cell viability. For the CCK- 8 assays, MIA PaCa-2 cells were seeded into a 96 -well culture plate $\left(3 \times 10^{3}\right.$ cells/well) in $200 \mu 1$ of complete medium. The plating medium was replaced with new culture medium after $24 \mathrm{~h}$. Then melatonin was dissolved in the new medium at different doses $(0.25-4 \mathrm{mM})$. Vehicle control cells were cultured in DMEM with $0.2 \%$ DMSO. Each group included three parallel wells. After exposurure for 12, 24, 48 and $72 \mathrm{~h}$, CCK- 8 was added to the culture media. The supernatant of each well was measured at a 450-nm wavelength using a plate reader (Infinite ${ }^{\circledR} 200$ PRO NanoQuant; Tecan Austria GmbH, Austria).

Wound-healing assay. MIA PaCa-2 cells were seeded into 12-well plates in complete medium at a density of $3 \times 10^{4}$ cells/well, at $37^{\circ} \mathrm{C}$ with $5 \% \mathrm{CO}_{2}$. When the cells formed a monolayer, a scratch was created in the middle of the well with a $100-\mu 1$ pipette tip. Subsequently, the debris was washed away and the wells were cultured with fresh media and $1 \%$ FBS with different concentrations of melatonin $(0-2 \mathrm{mM})$ and $0.2 \%$ DMSO. After incubation at 0,24 and $48 \mathrm{~h}$, images of the cells were captured (phase-contrast microscope). Each experiment was performed in triplicate. The initial migration of the scratch in the field of view was determined by the area divided by the length devoid of cells using Image-Pro Plus software (Media Cybernetics, Inc., Bethesda, MD, USA). Results are expressed as the difference in the migration distance between 0 and $48 \mathrm{~h}$ of treatment.

Apoptosis assay using Annexin V FITC/PI. To observe early apoptosis and necrosis, the cells were stained with FITC-conjugated Annexin V and propidium iodide (PI) (MultiSciences Biotech Co., Ltd., Hangzhou, China). The cells $\left(4 \times 10^{5}\right)$ were plated in 6 -well plates and treated with 0 and $2 \mathrm{mM}$ melatonin for 0 to $72 \mathrm{~h}$. Cells were harvested by trypsinization, cleared with PBS, centrifuged at $1,000 \mathrm{rpm}$ for $5 \mathrm{~min}$ and the supernatant was discarded. The pellet was resuspended in $1 \mathrm{X}$ binding buffer. A total of $500 \mu \mathrm{l}$ of the sample solution was added to $5 \mu \mathrm{l}$ of FITC-conjugated Annexin V and $10 \mu \mathrm{l}$ of PI and the solution was incubated for $5 \mathrm{~min}$ in the dark at room temperature. The cells were visualized and sorted using FACS (Becton Dickinson, San Jose, CA, USA) and quantified using FlowJo software. Positioning of quadrants on the Annexin V FITC/PI dot plots was used to distinguish living cells (Annexin V FITC'/ $\mathrm{PI}^{-}$), early apoptotic cells (Annexin $\mathrm{V} \mathrm{FITC} / \mathrm{PI}^{-}$) and late apoptotic/secondary necrotic cells (Annexin V FITC ${ }^{+} / \mathrm{PI}^{+}$) (18).

Western blot analysis. After $2 \mathrm{mM}$ melatonin treatment, the cells were washed three times with cold PBS and lysed by adding ice-cold RIPA buffer containing $50 \mathrm{mM}$ Tris- $\mathrm{HCl}$, $150 \mathrm{mM} \mathrm{NaCl}, 1 \%$ Triton $\mathrm{X}-100,1 \%$ sodium deoxycholate, $0.1 \%$ SDS, sodium orthovanadate, sodium fluoride, EDTA, leupeptin and PMSF and PhosSTOP (Roche, Basel, Switzerland) for 15 min on the table concentrator minus four degrees. Then cells were scraped off the plate. The extracts were transferred to a microfuge tube and centrifuged at $12,000 \mathrm{x} \mathrm{g}$ for $15 \mathrm{~min}$. The protein concentration was determined using the BCA assay (Beyotime). Equal amounts of total protein $(40 \mu \mathrm{g})$ were separately subjected to $10 \%$ SDS-PAGE and transferred to a PVDF membrane (Bio-Rad, Hercules, CA, USA). The membranes were blocked at room temperature for $1 \mathrm{~h}$ in blocking buffer and the proteins were incubated with primary antibodies targeted against: phospho-ERK $(1: 1,000)$, ERK $(1: 1,000)$, phospho-JNK (1:1,000), JNK (1:1,000), phospho-p65 $(1: 1,000)$, p65 (1:1,000) (Cell Signaling Technology, Beverly, MA, USA) and GAPDH $(1: 1,000)$ (Santa Cruz Biotechnology, Inc., Santa Cruz, CA, USA) for 12-16 h. After washing with TBST three times, the membranes were incubated for $1 \mathrm{~h}$ at room temperature with the secondary HRP-conjugated antibody (1:5,000; Bioworld Technology, Inc., USA) and visualized using WesternBright ECL detection kit (Advansta, Menlo Park, CA, USA). The density of the specific bands was quantified using Image Lab software with an imaging densitometer (Bio-Rad ChemiDoc MP) (both from Bio-Rad).

Statistical analysis. The results were analyzed using SPSS software (version 13) (SPSS, Inc., Chicago, IL, USA) and are presented as the mean values \pm SEM. Data comparisons were performed using analysis of variance (ANOVA). When the 


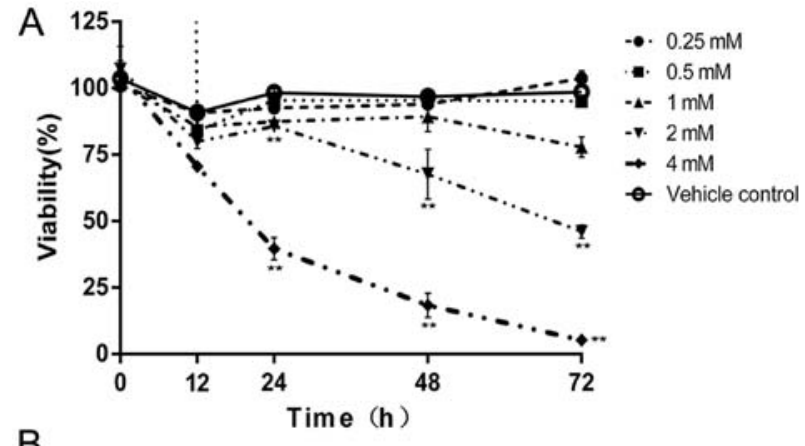

B

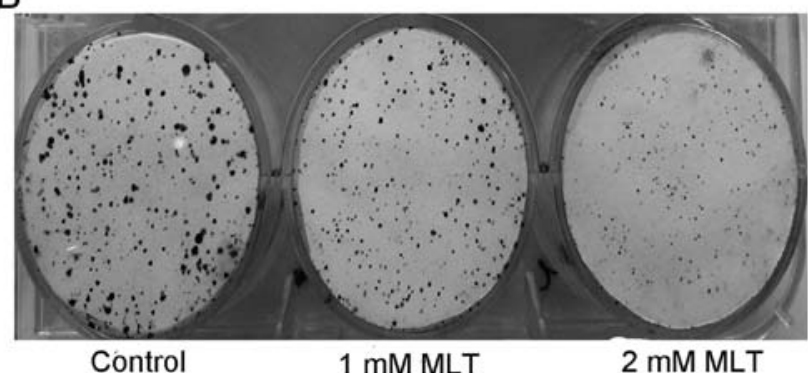

Figure 1. Effects of melatonin on the viability and colony formation of MIA PaCa-2 cells. (A) MIA PaCa-2 cells were treated for $0,12,24,48$ and $72 \mathrm{~h}$ with melatonin at varying concentrations $(0.25,0.5,1,2$ and $4 \mathrm{mM})$ and vehicle ( $0.2 \%$ DMSO). We used the CCK- 8 assay to assess MIA PaCa-2 cell viability. (B) MIA PaCa-2 cells were incubated in the absence (control) or presence of 1 and $2 \mathrm{mM}$ melatonin for 8 days. Treatment with melatonin inhibited colony formation in the MIA PaCa- 2 cells. The results are the means of three independent experiments. ${ }^{*} \mathrm{P}<0.05 ;{ }^{* * *} \mathrm{P}<0.01$ vs. the control group. MLT, melatonin.

analysis suggested the presence of a significant difference, the means were compared with the Newman-Keuls test. Statistical significance was accepted at $\mathrm{P}<0.05$.

\section{Results}

Melatonin affects MIA PaCa-2 cell viability, colony formation, invasion and apoptosis. We used the human pancreatic carcinoma cell line MIA PaCa-2 to evaluate the antitumour effects of melatonin on pancreatic cancer. The effect of melatonin on MIA PaCa- 2 cell viability was observed by the CCK- 8 assay and the results showed that cell viability was significantly reduced by 2 and $4 \mathrm{mM}$ melatonin after $12-72 \mathrm{~h}$ of treatment (Fig. 1A). When analysing the number of colonies formed, melatonin treatment (1 and $2 \mathrm{mM}$ ) caused a significant decline in colony formation (Fig. 1B).

Flow cytometry and Annexin V FITC/PI staining, which can identify early apoptotic cells, were used to assess apoptosis in the human pancreatic carcinoma cell line MIA PaCa-2 after exposure to $2 \mathrm{mM}$ melatonin for $0,24,48$ and $72 \mathrm{~h}$. As shown in Fig. 2A, after treatment for $48 \mathrm{~h}$ the percentage of early apoptotic cells was 1.79 -fold of that noted in the control cells. The early apoptotic rate (Annexin V FITC/PI staining) was significantly increased after $72 \mathrm{~h}$ (23.9\%, Fig. 2B).

MIA PaCa- 2 cells were treated with various concentrations of melatonin at various times to assess the effects of melatonin on cell migration. As shown in Fig. 3, 2 mM of melatonin markedly reduced MIA PaCa- 2 cell migration ( $20.1 \%$ of the control at $48 \mathrm{~h}$ ). To examine the inhibition of
A $0 \mathrm{~h}$

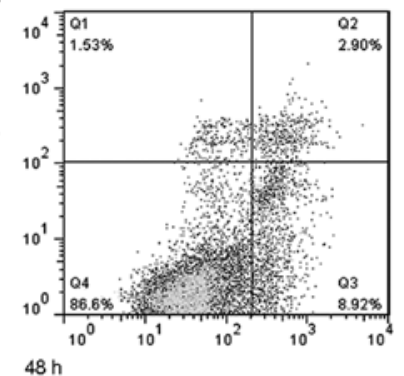

$24 \mathrm{~h}$
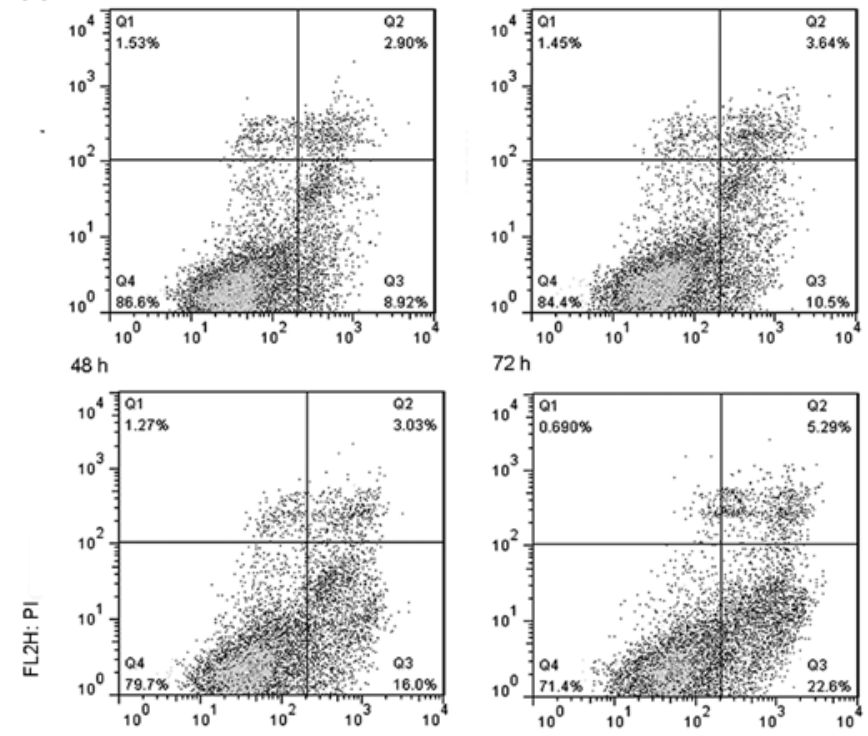

$72 \mathrm{~h}$

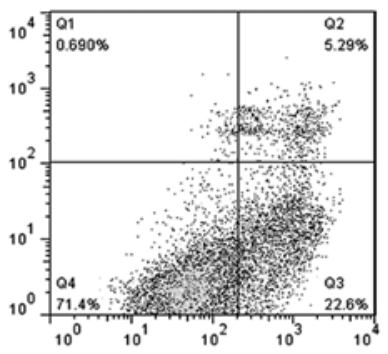

FL1H: Annexin V FITC

B

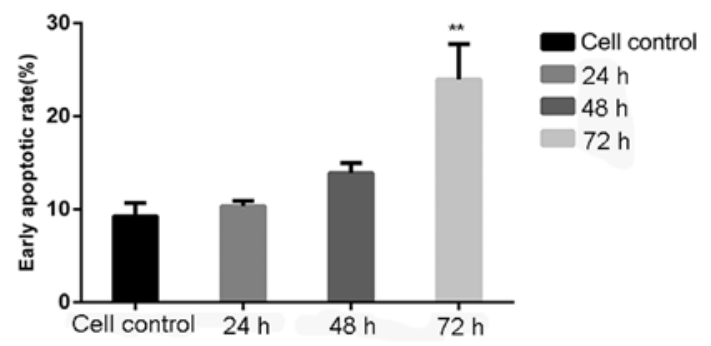

Figure 2. Melatonin promotes apoptosis of the MIA PaCa-2 cell line (A) MIA PaCa-2 cells were treated with $2 \mathrm{mM}$ melatonin for up to $72 \mathrm{~h}$. (B) The results are presented as the average early apoptotic rate from three randomized trials. Experimental data are presented as the means \pm SEM of three independent tests. ${ }^{*} \mathrm{P}<0.05 ;{ }^{* *} \mathrm{P}<0.01$ vs. control group $(0 \mathrm{~h})$.

cell motility by melatonin by invasion assay, we found that melatonin also suppressed cell invasion compared with the control group (Fig. 4).

Effects of melatonin on the phosphorylation of MAPK pathway components. Melatonin was found to inhibit cell viability and migration and induce cell apoptosis in the MIA PaCa- 2 cells. Considering the possible mechanisms, we evaluated the function on the elementary activation status of MAPKs. JNK and ERK phosphorylation was significantly induced at 24 and 48 h (Fig. 5A); the levels of JNK and ERK were used as internal controls and the phosphorylated proteins were quantified using the control. The results revealed that the levels of $\mathrm{p}$-JNK and p-ERK were increased at 24 and $48 \mathrm{~h}$ (Fig. 5B).

Melatonin inhibits $N F-\kappa B$ activation. Following treatment with $2 \mathrm{mM}$ melatonin, p65 phosphorylation was significantly induced in a time-dependent manner (Fig. 6A); the levels of p65 were used as internal controls and the phosphorylated proteins were quantified using the control. The results presented a clear decrease in p-p65 activity at 12 , 24 and 48 h (Fig. 6B). 
A
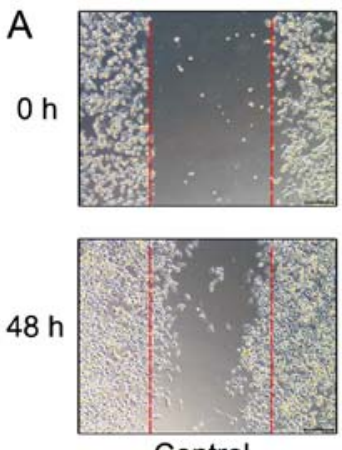

Control
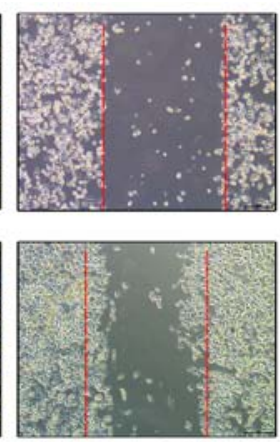

Vehicle control
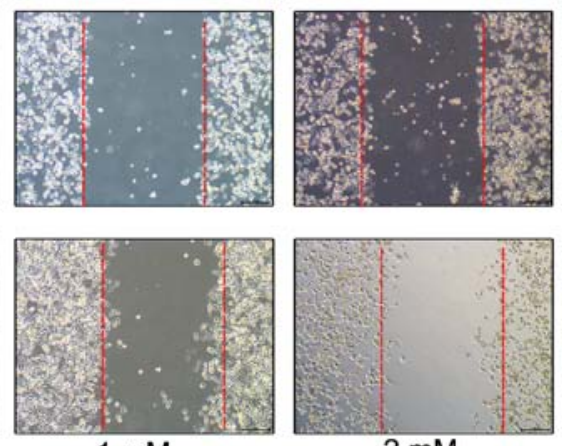

$1 \mathrm{mM}$

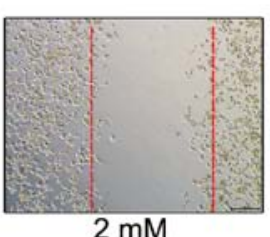

Melatonin

B

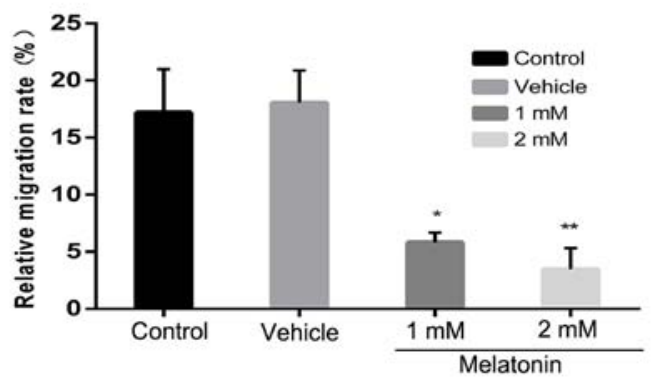

Figure 3. Melatonin inhibits the migration of the MIA PaCa-2 cell line. (A) Representative images of the migration of MIA PaCa-2 cells treated with 0-2 mM melatonin or vehicle at different time points $(0$ and $48 \mathrm{~h})$. (B) The relative migration rate at $48 \mathrm{~h}$ compared with the control cells. The data are from three independent experiments. ${ }^{*} \mathrm{P}<0.05 ;{ }^{* * *} \mathrm{P}<0.01$ vs. control group. MLT, melatonin.

A
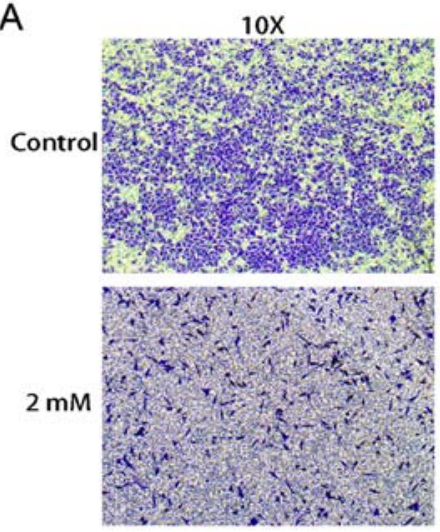

B

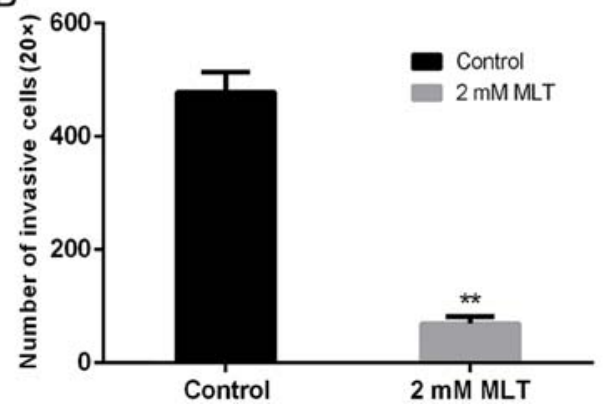

Figure 4. Melatonin inhibits the invasion of the MIA PaCa-2 cell line. (A) Representative images of the invasion of MIA PaCa-2 cells treated with 2 mM melatonin for $24 \mathrm{~h}$ (x10 and x20 magnification). (B) Analysis of the number of invasive cells treated with $2 \mathrm{mM}$ melatonin compared with the control cells. The data are from three independent experiments. ${ }^{*} \mathrm{P}<0.05 ;{ }^{* *} \mathrm{P}<0.01$ vs. control group. MLT, melatonin.

\section{Discussion}

Pancreatic cancer originates from pancreas tissue and has a high rate of metastasis (22). Pancreatic cancer is not only difficult to diagnose at an early stage, but also lacks effective therapeutic strategies (23). Recently, studies have revealed that melatonin has anticancer properties. However, the mechanisms of melatonin in regards to its antitumour properties are poorly 

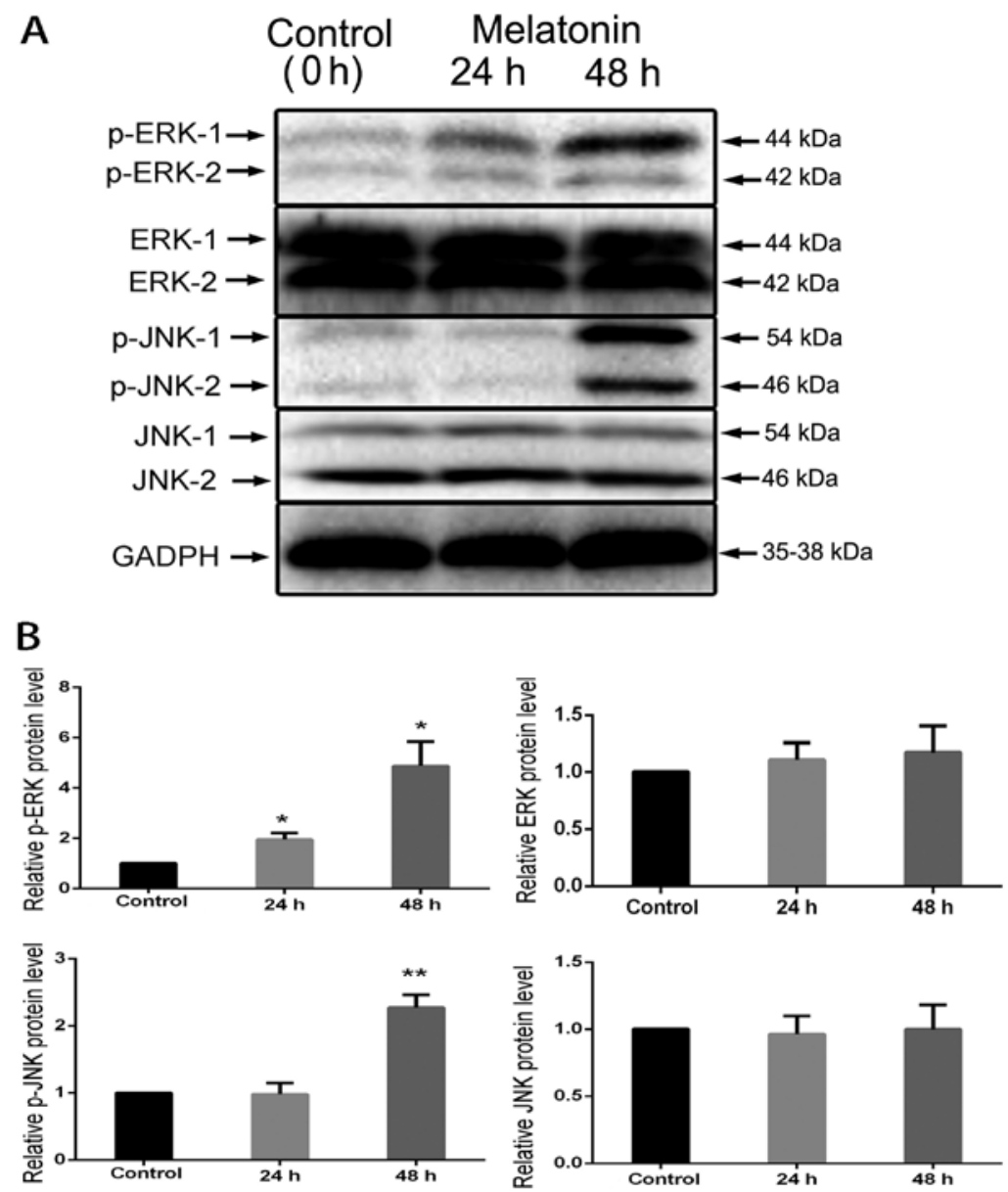

Figure 5. Effects of melatonin on the phosphorylation of MAPK pathway components. (A) MIA PaCa-2 cells were treated with $2 \mathrm{mM}$ melatonin for 0 to $48 \mathrm{~h}$ and the proteins were incubated with primary antibodies targeted against the following: anti-phospho-JNK, anti-JNK, anti-phospho-ERK and anti-ERK and assessed by western blot analysis. Protein signals were visualized with an ECL detection system. (B) The levels of JNK and ERK were used as internal controls. By densitometric analysis the phosphorylated proteins were quantified using the control. Experimental data are presented as the means \pm SEM of three independent experiments. ${ }^{*} \mathrm{P}<0.05 ;{ }^{* *} \mathrm{P}<0.01$ vs. control group. p-ERK, phospho-ERK; p-JNK, phospho-JNK.

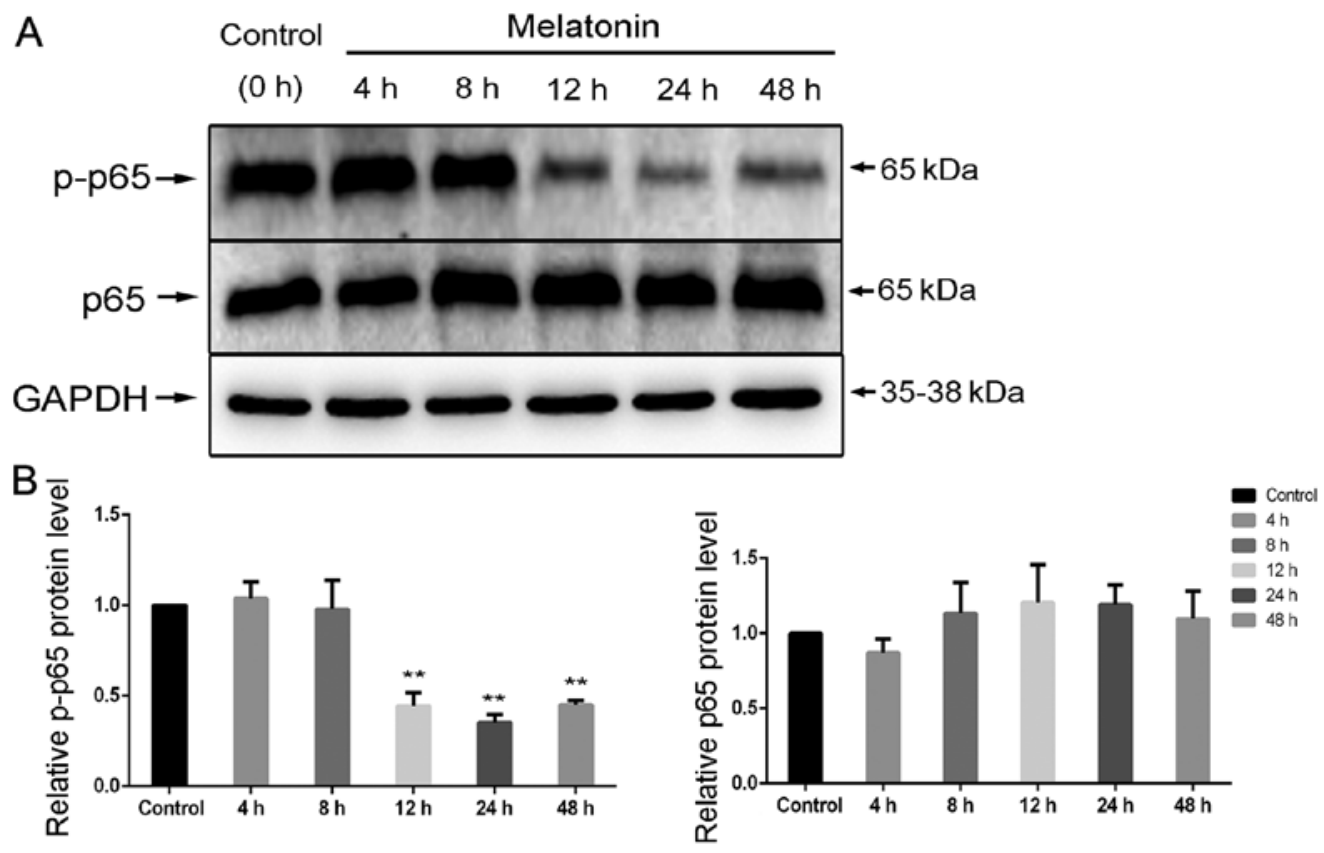

Figure 6. Melatonin inhibits NF- $\mathrm{kB}$ activation. (A) MIA PaCa-2 cells were treated with $2 \mathrm{mM}$ melatonin for 0 to 48 h. The proteins were incubated with primary antibodies targeted against: anti-phospho-p65, anti-p65 and GAPDH and assessed by western blotting analysis. Protein signals were visualized with an ECL detection system. (B) The levels of p65 were used as internal controls. By densitometric analysis the phosphorylated proteins were quantified using the control. Experimental data are presented as the means \pm SEM of three independent experiments. ${ }^{*} \mathrm{P}<0.05 ;{ }^{* *} \mathrm{P}<0.01$ vs. control group. p-p65, phospho-p65. 
understood. Induction of apoptosis is a potential antitumour mechanism and it is a basic step in the regulation of different cell types. Therefore, we studied melatonin which induced cell apoptosis in the human pancreatic carcinoma cell line MIA PaCa-2.

We found that melatonin inhibited MIA PaCa-2 cell viability, migration and invasion (Figs. 1, 3 and 4). Moreover, we revealed that melatonin induced cell apoptosis at various concentrations. Joo et al demonstrated that apoptosis activatescaspases which are related to cell viability (18). Pro-apoptosis is viewed as the most suitable strategy for treating cancer. Some reports suggest that ERK pathway activation influences a survival signal that weakens pro-apoptotic effects via activating JNK $(24,25)$. Cisplatin through ERK pathway activation, slows down cell growth and causes apoptotic cell death (26).

In summary, we considered whether melatonin through MAPK (JNK, ERK) pathways induces apoptosis in MIA $\mathrm{PaCa}-2$ cells. We hypothesized that melatonin acts as a potential apoptotic inducer. Our western blot analysis of MAPK pathway components showed that phosphorylation of JNK and ERK was enhanced at $24 \mathrm{~h}$ and significantly increased at $48 \mathrm{~h}$ by melatonin (Fig. 5). Recent research suggests an important role for JNK and ERK in pathways related to apoptosis and growth-inhibitory pathways $(27,28)$. In addition, JNK induces caspase-3 activation and JNK is necessary for the phosphorylation of proteins related to apoptosis in cancer cells, including Bcl-2 and Bax (29). In conclusion, we consider that melatonin caused MIA PaCa- 2 cell apoptosis by activating JNK and ERK which promote caspase-3 overexpression and caspase-3-induced cell apoptosis.

The transcription factor $\mathrm{NF}-\kappa \mathrm{B}$ family is comprised of related protein dimers (30). Upon activation, $N F-\kappa B$ p-65 is liberated from the I $\kappa \mathrm{B}$ compound and NF- $\kappa \mathrm{B}$ p- 65 translocates to the nucleus, where it causes the expression of a series of genes encoding different proteins which take part in suppressing apoptosis and causing inflammation, cellular invasion and proliferation (31). These target genes play key roles in malignant development and include apoptosis-suppressor proteins, such as Bcl-2 and Bcl-XL, and cell-cycle regulatory proteins, such as cyclin D1 (32). In malignant cells, stimulation of cell proliferation and protection against apoptosis are connected with abnormal NF- $\kappa$ B activation (33). Curcumin (34) and flavopiridol (35) have been suggested to inhibit NF- $\kappa \mathrm{B}$. In this study, we found that melatonin may suppress the phosphorylation of NF-кB p-65 (Fig. 6).

In summary, melatonin may possess anticancer effects in several types of cancer, including gastric cancer. Research suggests that melatonin exerts an antitumour effect (36). The potential to target mechanisms that inhibit NF- $\kappa \mathrm{B}$ p65 and promote ERK and JNK may offer effective strategies for chemotherapy. By analyzing the present and previous data, we established that melatonin induces apoptosis and suppresses migration and invasion via modulation of signaling mediated by the JNK and ERK MAPKs and NF- $\kappa$ B p65 pathways. The present study suggests the requirement for additional or adjunct therapies in combination with melatonin treatment to fully inhibit cancer progression. Our results suggest that melatonin may act as a potential anticancer agent against human pancreatic cancer.

\section{Acknowledgements}

This study was supported by the Science and Technology Bureau of Wenzhou,Zhejiang Province, China (no. 2014S0192).

\section{References}

1. Raimondi S, Maisonneuve P and Lowenfels AB: Epidemiology of pancreatic cancer: An overview. Nat Rev Gastroenterol Hepatol 6: 699-708, 2009

2. Guo L, Fan L, Pang Z, Ren J, Ren Y, Li J, Chen J, Wen Z and Jiang $X$ : TRAIL and doxorubicin combination enhances anti-glioblastoma effect based on passive tumor targeting of liposomes. J Control Release 154: 93-102, 2011.

3. Peixoto RD, Ho M, Renouf DJ, Lim HJ, Gill S, Ruan JY and Cheung WY: Eligibility of metastatic pancreatic cancer patients for first-line palliative intent nab-paclitaxel plus gemcitabine versus FOLFIRINOX. Am J Clin Oncol 1, 2015.

4. Conroy T, Desseigne F, Ychou M, Bouché O, Guimbaud R, Bécouarn Y, Adenis A, Raoul JL, Gourgou-Bourgade S, de la Fouchardière $\mathrm{C}$, et al; Groupe Tumeurs Digestives of Unicancer; PRODIGE Intergroup: FOLFIRINOX versus gemcitabine for metastatic pancreatic cancer. N Engl J Med 364: 1817-1825, 2011

5. Lerner AB, Case JD, Takahashi Y, Lee TH and Mori W: Isolation of melatonin, the pineal gland factor that lightens melanocytes. J Am Chem Soc 80: 2587-2587, 1958.

6. Berra B and Rizzo AM: Melatonin: Circadian rhythm regulator, chronobiotic, antioxidant and beyond. Clin Dermatol 27: 202-209, 2009.

7. Cardinali DP, Esquifino AI, Srinivasan V and PandiPerumal SR: Melatonin and the immune system in aging. Neuroimmunomodulation 15: 272-278, 2008.

8. Ambriz-Tututi M, Rocha-González HI, Cruz SL and Granados-Soto V: Melatonin: A hormone that modulates pain. Life Sci 84: 489-498, 2009.

9. Fischer TW, Kleszczyński K, Hardkop LH, Kruse N and Zillikens D: Melatonin enhances antioxidative enzyme gene expression (CAT, GPx, SOD), prevents their UVR-induced depletion, and protects against the formation of DNA damage (8-hydroxy-2'-deoxyguanosine) in ex vivo human skin. J Pineal Res 54: 303-312, 2013.

10. Shiu SY, Li L, Xu JN, Pang CS, Wong JT and Pang SF: Melatonin-induced inhibition of proliferation and G1/S cell cycle transition delay of human choriocarcinoma JAr cells: Possible involvement of MT2 (MEL1B) receptor. J Pineal Res 27: 183-192, 1999.

11. Xu L, Liu H, Zhang H, Wang RX, Song J and Zhou RX: Growth-inhibitory activity of melatonin on murine foregastric carcinoma cells in vitro and the underlying molecular mechanism. Anat Rec (Hoboken) 296: 914-920, 2013.

12. Xu L, Jin QD, Gong X, Liu H and Zhou RX: Anti-gastric cancer effect of melatonin and Bcl-2, Bax, p21 and p53 expression changes. Sheng Li Xue Bao 66: 723-729, 2014 (In Chinese).

13. Ordoñez R, Carbajo-Pescador S, Prieto-Dominguez N, García-Palomo A, González-Gallego J and Mauriz JL: Inhibition of matrix metalloproteinase-9 and nuclear factor kappa B contribute to melatonin prevention of motility and invasiveness in HepG2 liver cancer cells. J Pineal Res 56: 20-30, 2014.

14. Martín-Renedo J, Mauriz JL, Jorquera F, Ruiz-Andrés O, González P and González-Gallego J: Melatonin induces cell cycle arrest and apoptosis in hepatocarcinoma HepG2 cell line. J Pineal Res 45: 532-540, 2008.

15. Vijayalaxmi, Reiter RJ, Tan DX, Herman TS and Thomas CR Jr: Melatonin as a radioprotective agent: A review. Int J Radiat Oncol Biol Phys 59: 639-653, 2004.

16. Chang L and Karin M: Mammalian MAP kinase signalling cascades. Nature 410: 37-40, 2001.

17. Hsieh MH and Nguyen HT: Molecular mechanism of apoptosis induced by mechanical forces. Int Rev Cytol 245: 45-90, 2005.

18. Joo SS and Yoo YM: Melatonin induces apoptotic death in LNCaP cells via p38 and JNK pathways: Therapeutic implications for prostate cancer. J Pineal Res 47: 8-14, 2009.

19. Crawford LJ, Walker B and Irvine AE: Proteasome inhibitors in cancer therapy. J Cell Commun Signal 5: 101-110, 2011.

20. Tamura EK, Cecon E, Monteiro AWA, Silva CLM and Markus RP: Melatonin inhibits LPS-induced NO production in rat endothelial cells. J Pineal Res 46: 268-274, 2009. 
21. Gilad E, Wong HR, Zingarelli B, Virág L, O'Connor M, Salzman AL and Szabó C: Melatonin inhibits expression of the inducible isoform of nitric oxide synthase in murine macrophages: Role of inhibition of NFkappaB activation. FASEB J 12: 685-693, 1998

22. Hidalgo M, Cascinu S, Kleeff J, Labianca R, Löhr JM, Neoptolemos J, Real FX, Van Laethem JL and Heinemann V: Addressing the challenges of pancreatic cancer: Future directions for improving outcomes. Pancreatology 15: 8-18, 2015.

23. Xu B, Zhang K and Huang Y: Lin 28 modulates cell growth and associates with a subset of cell cycle regulator mRNAs in mouse embryonic stem cells. RNA 15: 357-361, 2009.

24. Hsiang C-H, Tunoda T, Whang YE, Tyson DR and Ornstein DK The impact of altered annexin I protein levels on apoptosis and signal transduction pathways in prostate cancer cells. Prostate 66: 1413-1424, 2006.

25. Mukherjee JJ, Gupta SK, Sikka H and Kumar S: Inhibition of benzopyrene-diol-epoxide (BPDE)-induced bax and caspase- 9 by cadmium: Role of mitogen activated protein kinase. Mutat Res 661: 41-46, 2009.

26. Sainz RM, Reiter RJ, Tan DX, Roldan F, Natarajan M, Quiros I, Hevia D, Rodriguez C and Mayo JC: Critical role of glutathione in melatonin enhancement of tumor necrosis factor and ionizing radiation-induced apoptosis in prostate cancer cells in vitro. J Pineal Res 45: 258-270, 2008.

27. Huang HL, Hsieh MJ, Chien MH, Chen HY, Yang SF and Hsiao PC: Glabridin mediate caspases activation and induces apoptosis through JNK1/2 and p38 MAPK pathway in human promyelocytic leukemia cells. PLoS One 9 :e98943, 2014.

28. Zhou Y, Zhao W, Xie G, Huang M, Hu M, Jiang X, Zeng D, Liu J, Zhou $\mathrm{H}$, Chen $\mathrm{H}$, et al: Induction of Nur77-dependent apoptotic pathway by a coumarin derivative through activation of JNK and p38 MAPK. Carcinogenesis 35: 2660-2669, 2014.
29. Liu J, Wu N, Ma L-N, Zhong JT, Liu G, Zheng LH and Lin XK: p38 MAPK signaling mediates mitochondrial apoptosis in cancer cells induced by oleanolic acid. Asian Pac J Cancer Prev 15: 4519-4525, 2014.

30. Aggarwal BB: Nuclear factor-kappaB: The enemy within. Cancer Cell 6: 203-208, 2004.

31. Deorukhkar A, Krishnan S, Sethi G and Aggarwal BB: Back to basics: How natural products can provide the basis for new therapeutics. Expert Opin Investig Drugs 16: 1753-1773, 2007.

32. Ahn KS and Aggarwal BB: Transcription factor NF-kappaB: A sensor for smoke and stress signals. Ann NY Acad Sci 1056: 218-233, 2005.

33. Yuan L, Wei S, Wang J and Liu X: Isoorientin induces apoptosis and autophagy simultaneously by reactive oxygen species (ROS)-related p53, PI3K/Akt, JNK, and p38 signaling pathways in HepG2 cancer cells. J Agric Food Chem 62: 5390-5400, 2014.

34. Bharti AC, Donato N, Singh S and Aggarwal BB: Curcumin (diferuloylmethane) down-regulates the constitutive activation of nuclear factor- $\kappa$ B and IkappaBalpha kinase in human multiple myeloma cells, leading to suppression of proliferation and induction of apoptosis. Blood 101: 1053-1062, 2003.

35. Takada Y and Aggarwal BB: Flavopiridol inhibits NF-kappaB activation induced by various carcinogens and inflammatory agents through inhibition of IkappaBalpha kinase and p65 phosphorylation: Abrogation of cyclin D1, cyclooxygenase-2, and matrix metalloprotease-9. J Biol Chem 279: 4750-4759, 2004.

36. Rondanelli M, Faliva MA, Perna S and Antoniello N: Update on the role of melatonin in the prevention of cancer tumorigenesis and in the management of cancer correlates, such as sleep-wake and mood disturbances: Review and remarks. Aging Clin Exp Res 25: 499-510, 2013. 